\title{
PROGNOSTIC SIGNIFICANCE OF MDM2 GENE EXPRESSION IN CHILDHOOD NEUROBLASTOMA
}

\author{
M.V. Inomistova ${ }^{*}$, N.M. Svergun ${ }^{1}$, N.M. Khranovska ${ }^{1}$, O.V. Skachkova ${ }^{1}$, O.I. Gorbach ${ }^{1}$, G.I. Klymnyuk ${ }^{2}$ \\ ${ }^{1}$ Laboratory of Experimental Oncology, National Cancer Institute, Kyiv 03022, Ukraine \\ ${ }^{2}$ Pediatric Oncology Department, National Cancer Institute, Kyiv 03022, Ukraine
}

\begin{abstract}
Aim: To investigate the association of MDM2 expression at the mRNA levels in neuroblastoma with clinical features and unfavorable disease factors to determine the possibility of it usage as a prognostic marker of neuroblastoma. Materials and Methods: Total RNA and DNA were extracted from tumor tissue samples of total 91 neuroblastoma patients (mean age: $39.45 \pm 4.81$ months). MDM2 mRNA levels were detected with Q-PCR. TP53 gene deletion was detected with FISH method. MYCNamplification was detected with Q-PCR analysis in fresh tumor samples and FISH in FFPE samples. Results: We investigated the association of MDM2 mRNA expression with clinical outcome in neuroblastoma patients $(\mathrm{n}=91)$. Kaplan - Meier curves showed a significant association of high $M D M 2 \mathrm{ex}-$ pression with poor event-free survival $(\mathrm{p}<0.001)$. Clinical outcome of patients without $M Y C N$ amplification with low $M D M 2$ expression was associated with better event-free survival than with high $M D M 2$ expression $(\mathrm{p}<0.001)$. Overexpression of $M D M 2$ can be used as significant prognostic marker for patient stratification on risk groups and treatment optimization. Conclusion: Our results showed that the high expression of MDM2 at mRNA levels is an important factor of neuroblastoma prognosis. It may be a valuable additional molecular marker in guiding specific therapy in MYCN non-amplified NB patients without TP53 gene deletion.

Key Words: neuroblastoma, MDM2 mRNA expression, MYCN gene amplification, p53/MDM2 interaction.
\end{abstract}

Neuroblastoma (NB) is an embryonal tumor arising in tissues of the sympathetic nervous system and the most common cancer diagnosed during the first year of life. NB accounts for $15 \%$ of all deaths due to childhood malignancies. Despite intense multimodality therapy, at least half of high-risk patients relapse that is almost always fatal [1]. Much of the difficulty in devising effective therapies for this latter group of patients lies in the heterogeneity of disease, which can be attributed largely to the interaction of multiple genetic and epigenetic factors [2].

Unlike many adult malignancies most NB do initially respond to chemotherapy and it is likely that the presence of functionally active $p 53$ is at least partly involved in this initial chemosensitivity. However, over half of previously responsive NB eventually relapses with chemoresistant disease and there is evidence for $\mathrm{p} 53$ inactivation at this stage in some cases [3]. p53 is a potent transcription factor that modulates a large number of genes involved in apoptosis, metabolism, epigenetics, and cell cycle regulation [4]. p53 is usually expressed at low levels in the cell where it has a short half-life of only $30 \mathrm{~min}$ [5]. NB is a p53 wild type malignancy at diagnosis and repression of p53 signaling plays an important role in its pathogenesis. Chemotherapy induces apoptosis and tumor regression primarily through activation of p53mediated transcription. A number of previous observations confirm that wild-type p53 alleles are present in the vast majority of cases of newly diagnosed NB, but

\section{Submitted: December 18, 2014. \\ ${ }^{\star}$ Correspondence: $\quad$ E-mail: m.inomistova@gmail.com Tel.: +38(093)505-56-99}

Abbreviations used: ARF - adenosine diphosphate-ribosylation factor; a.u. - arbitrary units; AUC - area under the curve; FFPE formalin-fixed, paraffin embedded tissue; FISH - fluorescence in situ hybridization; MNA - MYCN gene amplification; NB - neuroblastoma; PCR - polymerase chain reaction. that p53/MDM2/adenosine diphosphate-ribosylation factor (ARF) responses to chemotherapy are repressed, in part due to unscheduled inhibition of p53 by MDM2. This suggests that downregulation of the p53 axis may underlie the treatment resistance typically seen in highrisk NB [4]. The expression, function and stabilization of p53 are governed by a complex network, which includes its regulators p14ARF and MDM2 [5].

Originally identified as an amplified gene located on a double minute chromosome in a transformed mouse cell line, MDM2 was later shown to have a critical role in the process of cellular transformation. Thus, deregulation of $M D M 2$ gene expression likely contributes to the pathogenesis of a wide range of human tumors. Amplification and/or overexpression of MDM2 is found in about $10 \%$ of all human tumors. In gliomas, for example, MDM2 amplification identifies a subset of high-risk patients that do not have p53 mutations [6]. In many soft tissue sarcomas, MDM2 amplification and overexpression correlates with poor prognosis.

Additionally, p53 activates the MDM2 gene that encodes p53 antagonist forming an autoregulatory feedback loop that tightly controls expression of both p53 and MDM2 [7]. MDM2 helps to target p53 for degradation through its E3 ubiquitin ligase activity.

Recent literature demonstrates that p53 is generally functional, accumulates in the nucleus in response to DNA damage and is efficiently degraded by MDM 2 in NB cells. There are reports that 553 function may be compromised as a consequence of aberrant MDM2 expression levels in NB. For instance, a study examining etoposide-induced p53 activity in a NB cell lines suggested an important role for elevated MDM2 expression levels in the regulation of p53 nuclear-cytoplasm translocation [8].

Also, elevated MDM2 expression and accompanying loss of p53 function is associated with multidrug 
resistance in some NB cell lines. MDM2 ubiquitin ligase activity is rate-limiting for p53 degradation and is critical in NB [9]. Taken together, these data demonstrate that the MDM2/p53 interaction is intact in NB and suggest that deficiencies in p53 functions may be a consequence of aberrant MDM2 expression.

Amplification of the MYCN gene (MNA) has emerged as one of the most reliable indicators of aggressive and treatment-resistant NB, yet $30 \%$ to $40 \%$ of high-risk tumors lack this feature [1]. MNA is associated with an exceptionally poor prognosis in NB. Furthermore, the crucial effectors of $M Y C N$ responsible for this aggressive subset of NB await characterization [8]. MYC oncogenes activate both proliferative and apoptotic cellular pathways and, accordingly, inhibition of p53-mediated apoptosis is a prerequisite for MYC-driven tumorigenesis [10]. A critical negative regulator of the p53 tumor suppressor, MDM2, has been recently characterized in NB cell lines as a transcriptional target of MYCN. Targeted inhibition of $M Y C N$ results in reduced $M D M 2$ expression levels, with concomitant stabilization of p53 and stimulation of apoptosis in MNA NB cell lines [8]. Moreover, $M D M 2$ has a p53-independent role in the regulation of both MYCN mRNA stabilization and its translation, suggesting that MDM2-mediated MYCN expression is one mechanism associated with growth of $M Y C N$ associated NB and disease progression [11]. MYCN also transcriptionally upregulates $p 53$ expression in NB and may be an important mechanism by which MYCN induces apoptosis [12]. Scheme of MDM2 and p53 regulation involving MYCN is shown in Fig. 1.

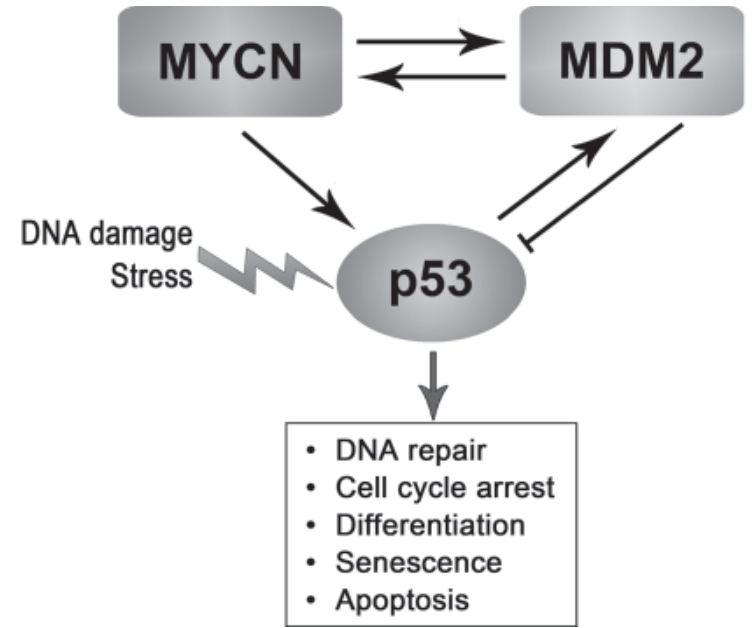

Fig. 1. Scheme of MDM2 and p53 regulation involving $M Y C N$ oncogene

In this article, we have investigated the association of MDM2 mRNA expression in NB with clinical characteristics and unfavorable disease factors (occurrence age, disease stage, MYCN gene status, metastases and relapses) to determine the possibility of it usage as a prognostic marker of NB.

\section{MATERIALS AND METHODS}

Patients. 91 patients with verified diagnosis of NB have been enrolled into this study. The age of patients ranged from 1 month to 17 years. Mean age was $39.45 \pm 4.81$ months. Age of $28.6 \%$ patients did not exceed 18 months. Stages distribution: I-II stage $21.25 \%$ of patients, III stage $-22.5 \%$ of patients, IV stage $-56.25 \%$ of patients. All patients were treated in accordance with international standards of treatment for NB. Informed consent was obtained according to the requirements of the Ethics Commission of the National Cancer Institute.

Material. Tumor tissue material was obtained by biopsy or tumor resection. $87.9 \%$ of samples were obtained from primary NB tumors, $4.4 \%$ - from recurrent tumors, $7.7 \%$ - from metastatic focus. All biomaterial was taken before treatment. Fresh tumor samples for Q-PCR analysis were stored in "RNA-later" ("Ambion", USA) to stabilize the RNA and DNA; for fluorescent in situ hybridization (FISH) formalin-fixed, paraffin embedded tissue (FFPE) samples were used.

Analysis of TP53 gene deletion. Deletion of TP53 gene was investigated in NB tumors using FISH analysis. Preparation of FFPE tissue sample slides was performed using Paraffin Pretreatment Reagent Kit I (Abbott, USA). FISH analysis was performed using dual-color Vysis LSI p53 (17p13.1)/CEP17 Probe (Abbott, USA). Analysis of the hybridization was carried out using a fluorescence microscope Axio Imager M1 (Zeiss, Germany).

Analysis of MNA. Determination of MYCN gene status was assessed in NB fresh tumors by Q-PCR analysis and FISH for FFPE samples. Genomic DNA from tumor samples was isolated using "NucleoSpin Tissue" (Macherey-Nagel, Germany). Sequences of primers were experimentally selected with Primer Express ${ }^{\circledR}$ Software v3.0 (Applied Biosystems, USA) and synthesized by "Applied Biosystems", they are shown in the Table 1. Primers and TaqMan probes were used at $0.6 \mu \mathrm{M}$ and $0.4 \mu \mathrm{M}$ concentrations, respectively. TaqMan Universal PCR Master Mix (Applied Byosystems, USA) and $5 \mathrm{ng}$ of DNA were used to prepare the reaction mixture in a total volume of $25 \mu \mathrm{l} .45$ cycles real-time PCR $\left(94^{\circ} \mathrm{C}-3 \mathrm{sec}, 60{ }^{\circ} \mathrm{C}-30 \mathrm{sec}\right.$ and $72^{\circ} \mathrm{C}-30 \mathrm{sec}$ ) were run on $7300 / 7500$ Real-Time PCR Systems, "Applied Biosystems", USA. MYCN gene copy number was calculated by the formula:

$$
x=2^{-\Delta C t},
$$

where $x-M Y C N$ copy number,

$\Delta \mathrm{Ct}=\mathrm{Ct}(\beta$-actin $)-\mathrm{Ct}(M Y C N)$.

$\mathrm{FISH}$ analysis was performed using dual-color Vysis LSI $^{\circledR} \mathrm{N}-\mathrm{MYC}$ Spectrum Green/CEP 2 Spectrum Orange ${ }^{\mathrm{TM}}$ (Abbott, USA). According to the European Network for Quality Assurance in Higher Education guidelines [13], MNA was defined as $>4$-fold increase of $M Y C N$ signal number in relation to the number of chromosome 2 .

\begin{tabular}{|c|c|}
\hline$\beta$-actin & F: 5'TCACCCACACTGTGCCCATCTACGA3' \\
\hline MDM2 & $\begin{array}{l}\text { F: } 5^{\prime} \text { CGACTCCAAGCGCGAAA3' } \\
\text { B. } 3^{\prime} \text { GGTICAGCACCATCAGTGGTACA }\end{array}$ \\
\hline GADPH & $\begin{array}{l}\text { F: 5'TCACCCACACTGTGCCCATCTACGA3' } \\
\text { R: } 3 \text { 'CAGCGGAACCGCTCATTGCCAATGA5' }\end{array}$ \\
\hline
\end{tabular}


Detection of MDM2 mRNA expression level.

RNA was isolated from tumor tissue using "Total Nucleic Acid Isolation Kit" (Ambion, USA). Reverse transcription was conducted using PCR test set "High Capacity cDNA Reverse Transcription Kit" (Applied Biosystems, USA) in a total volume of $20 \mu \mathrm{l}$ with $1 \mu \mathrm{l}$ of total RNA. The reverse transcription reaction was run under the following conditions: $25^{\circ} \mathrm{C}-10 \mathrm{~min}, 37^{\circ} \mathrm{C}-120 \mathrm{~min}$ and $85^{\circ} \mathrm{C}-5 \mathrm{sec}$. GADPH was used to normalize levels of $\mathrm{mRNA}$ for the relative quantification method of analysis. Sequences of primers (Applied Biosystems, USA) are shown in the Table 1. Primers and TaqMan probes were used at $0.3 \mu \mathrm{M}$ and $0.2 \mu \mathrm{M}$ concentrations, respectively. PCR reaction was performed in accordance with such as in the detection of MNA. Calculations were performed using the $\Delta \triangle$ Ctrelative quantification method. The thresholds and baselines were set manually in SDS and Ct values were extracted.

Statistical analysis. All experiments were repeated independently at least three times. Gaussian distribution of the group was checked with Kolmogorov - Smirnov tests. Analysis of the association between the studied markers and clinical characteristics of patients was performed using U-Mann - Whitney test. Prognostic significance of markers was verified with the ROC-curve (Receiver Operating Characteristic curve). Event free survival was evaluated by Kaplan - Meier estimator, statistical significance of parameters differences was determined using F-Cox criterion. The impact of gene expression in NB patients' survival was evaluated using the Cox proportional hazards regression analysis [14]. The data were processed with the software package Statistica 6.1 using parametric and nonparametric methods of statistical analysis and MedCalc 12.1.4.0. The difference was considered statistically significant at $p<0.05$.

\section{RESULTS AND DISCUSSION}

Mutations in the TP53 gene rarely are observed in childhood cancer of central and peripheral nervous system. In primary NB tumors frequency of its mutation does not exceed $1-2 \%$ and $15 \%$ in recurrent and/ or progressive tumors. Loss of 17p chromosome region, mapping TP53 gene, is more usual for NB [4]. In our study no tumor sample with $17 p$ chromosome region deletion was found in patients with NB. But we suggest that deficiencies in p53 functions may be a consequence of aberrant MDM2 expression showed further.

Patients were dichotomized by age into patients younger than 18 months and patients 18 months of age and older. Stage was dichotomized for the entire NB cohort with respect to metastatic progression (i.e., stage I, II, and III vs stage IV). MNA was found in $27.5 \%$ NB patients. These patients were classified as high risk group and treated with the relevant protocols. The average age of patients with MNA was $29.91 \pm 3.59$ months. Among all patients with MNA $36 \%$ of patients were with localized disease stages, $64 \%$ - IV stage. The frequency of MNA detected in our study (27.5\%) did not differ from the data obtained by other research groups [2].

We have investigated whether MDM2 overexpression was associated with various clinical features (Table 2 ).
Table 2. Clinical and biological characteristics and MDM2 mRNA expression in NB tumor samples

\begin{tabular}{|c|c|c|c|c|}
\hline № & \multicolumn{3}{|c|}{ Clinical and biological features } & \multirow{2}{*}{$\begin{array}{c}\text { MDM2 mRNA } \\
\text { expression } \\
\text { level, a.u. } \\
1.29 \pm 0.29 \\
41.116 \pm 25.88^{1} \\
3.36 \pm 0.99^{1}\end{array}$} \\
\hline 1 & $\begin{array}{l}\text { Biological } \\
\text { material }\end{array}$ & $\begin{array}{l}\text { Primary tumor } \\
\text { Recurrent tumor } \\
\text { Metastatic foci }\end{array}$ & $\begin{array}{c}\mathrm{n}=80(87.9 \%) \\
\mathrm{n}=4(4.4 \%) \\
\mathrm{n}=7(7.7 \%)\end{array}$ & \\
\hline & & Primary $\mathrm{t}$ & mors: & \\
\hline 2 & Age $(n=79)$ & $\begin{array}{l}\text { Under } 18 \text { months } \\
\text { Over } 18 \text { months }\end{array}$ & $\begin{array}{l}\mathrm{n}=29(36.7 \%) \\
\mathrm{n}=50(63.3 \%)\end{array}$ & $\begin{array}{l}0.82 \pm 0.30 \\
1.58 \pm 0.43\end{array}$ \\
\hline 3 & Stage $(n=69)$ & & $\begin{array}{l}n=32(46.4 \%) \\
n=37(53.6 \%)\end{array}$ & $\begin{array}{c}0.72 \pm 0.31 \\
2.06 \pm 0.54^{2}\end{array}$ \\
\hline 4 & $\begin{array}{l}\text { MYCN gene } \\
\text { status }(\mathrm{n}=78)\end{array}$ & $\begin{array}{l}\text { Non-amplified } \\
\text { Amplified }\end{array}$ & $\begin{array}{l}\mathrm{n}=56(71.8 \%) \\
\mathrm{n}=22(28.2 \%)\end{array}$ & $\begin{array}{l}0.86 \pm 0.2 \\
2.47 \pm 0.9^{3}\end{array}$ \\
\hline
\end{tabular}

Note: a.u. - arbitrary units; ${ }^{1} p<0.001-$ significant regarding primary tumors; ${ }^{2} p<0.001$ - significant regarding I-III stages; ${ }^{3} p<0.03$ - significant regarding $M Y C N$ non-amplified.

MDM2 mRNA expression level was significantly higher in recurrent tumors compared to primary tumors and metastatic sites $(p<0.001)$. Primary tumors were characterized by the lowest level of MDM2 expression. These data do not contradict the findings of other research groups [15], and can testify in favor of the theory that the inactivation of $\mathrm{p} 53$ by MDM2 in NB often develops during treatment and may be involved in the progression and metastasis of the disease and the occurrence of relapses.

In primary tumors the value of MDM2 mRNA expression varied quite widely, especially depending on the disease stage and MYCN gene status. MDM2 expression level was significantly higher in tumor samples obtained from patients with disseminated disease stage compared with early stages and in patients with MNA compared with $M Y C N$-negative tumors. Thus, higher MDM2 expression was associated with unfavorable clinical NB features (MNA, disease stage), indicating a link between MDM2 overexpression and the high-risk phenotype.

We conducted ROC-analysis to assess the prognostic significance of $M D M 2$ mRNA expression in NB progression. ROC-analysis is one of the most accurate statistical methods to assess the diagnostic and prognostic significance of the marker. For patients distribution on groups according to MDM2 mRNA expression level we applied event free survival rate as the criterion of clinical efficacy. As a result, optimal criterion for definition of high and low MDM2 mRNA expression level was established, it was $>0.0822$ a.u. It was found that the area under the curve (AUC) was $0.75(p=0.001)$. According to the ROC-analysis (Fig. 2) high MDM2 expression was shown as a marker of unfavorable NB and risk of disease recurrence. This marker had sufficient sensitivity and specificity and may serve as an independent marker of prognosis and stratification of NB patients on risk groups.

To confirm obtained results we have analyzed the event free survival of NB patients depending on MDM2 mRNA expression level. With this aim all patients were divided into 2 groups according to MDM2 expression level (high and low expression) under ROCanalysis optimal criterion (Fig. 3). 


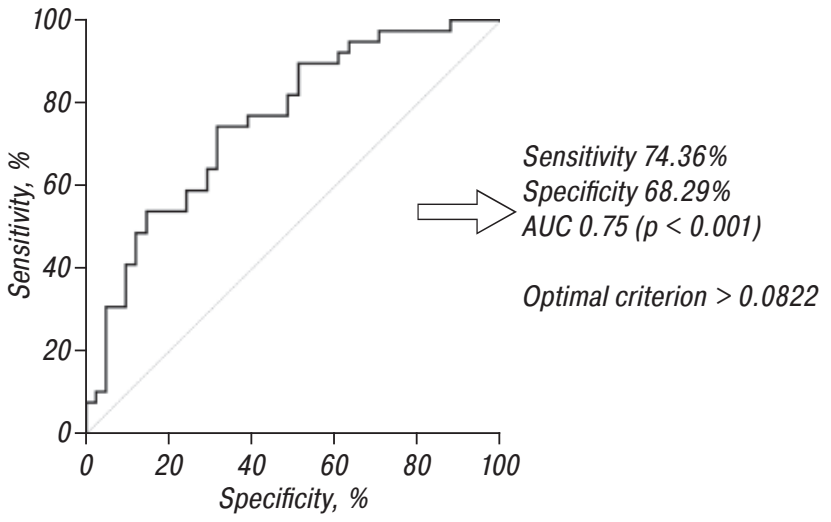

Fig. 2. ROC curve for $M D M 2 \mathrm{mRNA}$ expression ratio in primary NB

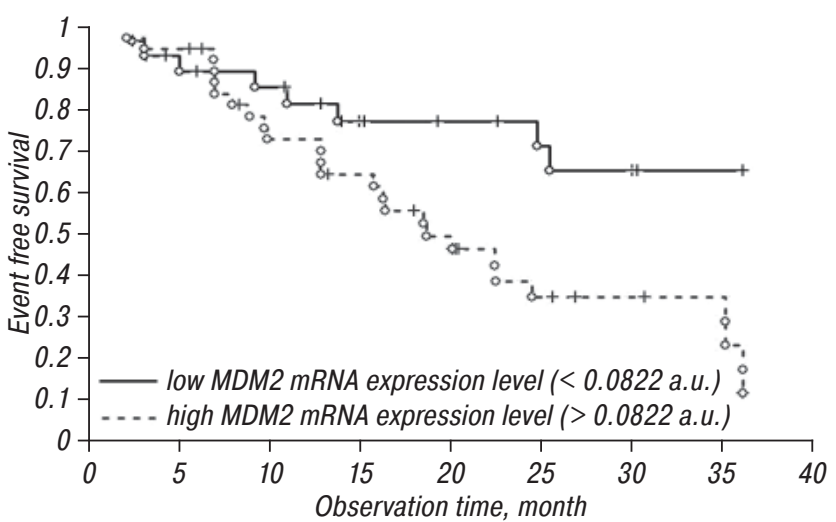

Fig. 3. Kaplan - Meier event free survival curves according to optimal criterion of MDM2 mRNA expression level in primary NB tumors $(p<0.001)$

As the result, overexpression of the MDM2 was found in $52.5 \%(42 / 80)$ of patients. We have found that the high level of MDM2 expression was associated with a significant decrease in event free survival of NB patients, regardless $M Y C N$ gene status and disease stage $(p<0.001)$. Accordingly, the rate of 3-year event free survival of patients with MDM2 mRNA high expression was only $12 \%$, whereas patients with low expression $66 \%$. Such a significant difference indicates that this marker has substantial role in the pathogenesis and progression of NB and MDM2/p53 imbalance leads to poor prognosis in patients. As follows, along with other clinical features it can be used as prognostic and possibly therapeutic approach for treatment optimization. In a Cox proportional hazards model of the risk factors, high MDM2 expression was an independent factor for event free survival of NB patients $(p=0.002$, $\mathrm{Cl} 95 \%$ 1.06-1.30). Therefore, raised MDM2 expression level is associated with the risk of disease recurrence.

Since MYCN oncogene transactivates MDM2 [8, 11], in NB with MNA, increased expression/activity of MDM2 could significantly decrease the activity of p53, resulting in failure to undergo appropriate cell cycle arrest and/or apoptosis. We have analyzed changes of MDM2 expression and event free survival in tumors with different $M Y C N$ gene status.

In determining of optimal criterion for MDM2 mRNA expression level by ROC-analysis in MNA group no precise parameter for distribution on risk groups was established. With Cox regression analysis we have found that changes in MDM2 expression level in this group does not affect event free survival ( $p=0.14, \mathrm{Cl} 95 \%$ 0.97-1.26). Changes of MDM2 mRNA expression in this group can be explained by MDM2/p53 interaction malfunction caused by different reasons. Also they may be caused by MNA activating effect on MDM2 and MDM2-mediated $M Y C N$ expression promotion. In any case, this leads to poor prognosis; disease progression that often occurs even in patients with MNA and low MDM2 gene expression, indicating the complexity of NB biology.

Particular attention of oncologists and scientists attracts MYCN non-amplified NB, as it has yet to be known factors that lead to rapid progression and aggressive course of the disease in some patients or spontaneous regression and favorable prognosis in others. For this NB group $(n=56)$ with ROC-analysis we have established the possibility of MDM2 mRNA expression using as prognostic marker and have identified the optimal criterion for distribution on favorable/unfavorable prognostic groups (Fig. 4).

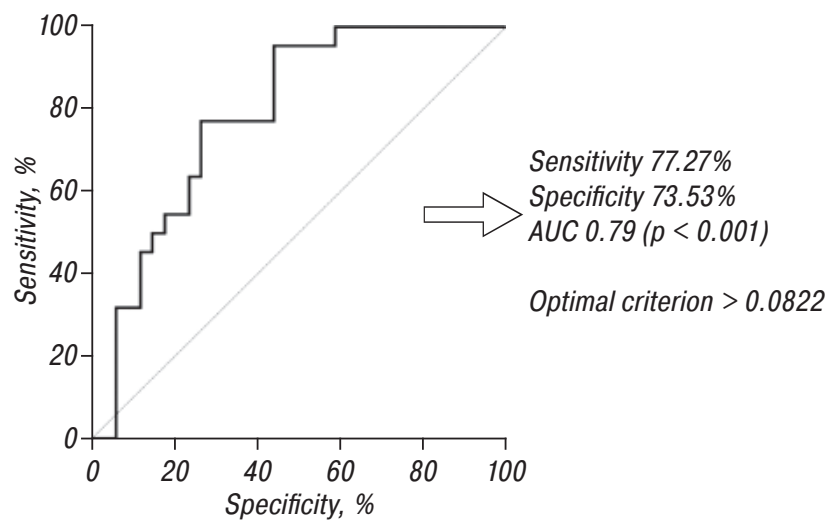

Fig. 4. ROC curve for MDM2 mRNA expression ratio in $M Y C N$ non-amplified NB

Finally, we investigated the prognostic value of MDM2 expression in this group. Kaplan - Meier survival curves revealed that the clinical outcome (event free survival) was on 64\% higher in low MDM2 expression group compared to high MDM2 expression group, $\mathrm{p}<0.001$ (Fig. 5). Higher than optimal criterion expression level was detected in $46.4 \%$ tumor samples. With Cox regression model we have found that changes in MDM2 mRNA expression level in MYCN non-amplified NB predetermines event free survival $(p<0.05, \mathrm{Cl} 95 \%$ 1.004-1.59).

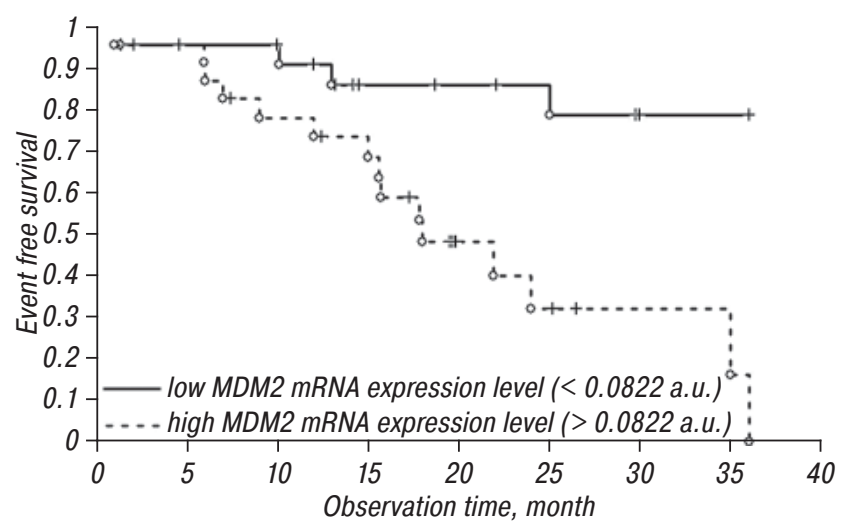

Fig. 5. Kaplan - Meier event free survival curves according to optimal criterion of MDM2 mRNA expression level in MYCN non-amplified NB $(p<0.001)$ 
These data suggest that $M D M 2$ can be useful in prediction of NB prognosis and outcome. In MYCN nonamplified NB regulatory contribution of $M Y C N$ is offset. In this group, deterioration in disease course of the patient with high MDM2 mRNA expression is directly connected with dysregulation in MDM2/p53 interaction.

\section{CONCLUSIONS}

Our results have shown that MDM2 mRNA expression is an important factor in NB prognosis. For patients that do not show chromosomal rearrangements of TP53 gene locus, elevated expression of MDM2 mRNA can serve as an effective and useful prognostic marker under certain circumstances. Thus, when MDM2 is expressed at the high level in a group with MNA, this has a reinforcing effect on the progression of the disease. Elevated levels of MDM2 in NB without MNA might be used as the significant prognostic marker for both, patient stratification on risk groups and for treatment optimization.

\section{REFERENCES}

1. Maris JM. Recent advances in neuroblastoma. N Engl J Med 2010; 362: 2202-11.

2. Cohn SL, Pearson AD, London WB, et al. The International Neuroblastoma Risk Group (INRG) classification system: an INRG Task Force report. J Clin Oncol 2009; 27: 289-97.

3. Tweddlea DA, Pearson AD, Haber M, et al. The p53 pathway and its inactivation in neuroblastoma. Cancer Lett 2003; 197: 93-8.

4. Barbieri E, De Preter K, Capasso M, et al. A p53 drug response signature identifies prognostic genes in high-risk neuroblastoma. PLOS one 2013; 8: 1-11.
5. Barone G, Tweddle DA, Shohet JM, et al. MDM2p53 interaction in paediatric solid tumours: preclinical rationale, biomarkers and resistance. Cur Drug Targ 2014; 15: $114-23$.

6. Reifenberger G, Liu L, Ichimura K, et al. Amplification and overexpression of the MDM2 gene in a subset of human malignant gliomas without p53 mutations. Cancer Res 1993; 53: $2736-9$.

7. Deb SP. Cell cycle regulatory functions of the human oncoprotein MDM2. Mol Cancer Res 2003; 1: 1009-16.

8. Slacka A, Lozanob G, Shohet JM. MDM2 as MYCN transcriptional target: Implications for neuroblastoma pathogenesis. Cancer Lett 2005; 228: 21-7.

9. Isaacs JS, Saito S, Neckers LM. Requirement for HDM2 activity in the rapid degradation of p53 in neuroblastoma. J Biol Chem 2001; 276: 18497-506.

10. Slack A, Chen Z, Tonelli R, et al. The p53 regulatory gene MDM2 is a direct transcriptional target of MYCN in neuroblastoma. Proc Natl Acad Sci USA 2005; 102: 731-6.

11. Gu L, Zhang H, He J, et al. MDM2 regulates MYCN mRNA stabilization and translation in human neuroblastoma cells. Oncogene 2012; 31: 1342-53.

12. Chen L, Iraci N, Gherardi S, et al. p53 is a direct transcriptional target of MYCN in neuroblastoma. Cancer Res 2010; 70: 1377-88.

13. Ambros PF, Ambros IM. SIOP Europe Neuroblastoma Pathology, Biology and Bone Marrow Group. Pathology and biology guidelines for resectable and unresectable neuroblastic tumors and bone marrow examination guidelines. Med Pediatr Oncol 2001; 37: 492-504.

14. Kalbfleisch JD, Prentice RL. The statistical analysis of failure time data. New York: John Wiley \& Sons, 2002.462 p.

15. Cattelani S, Ferrari-Amorotti G, Rachele Soliera AR, et al. Neuroblastoma: role of MDM2 and SNP309 as markers. Ped Cancer 2013; 4: 19-25. 